\title{
Rules-plus-exception tasks: A problem for exemplar models?
}

\author{
Pedro M. Rodrigues and JaAP M. J. Murre \\ University of Amsterdam, Amsterdam, The Netherlands
}

\begin{abstract}
Erickson and Kruschke (2002b) have shown that human subjects generalize category knowledge in a rulelike fashion when exposed to a rule-plus-exception categorization task. This result has remained a challenge to exemplar models of category learning. We show that these models can account for such performance, if they are augmented with exemplar-specific specificity or exemplar-specific attention. This result, however, is only achieved if the choice rule that converts evidence for competing categories into probabilities is sensitive to small differences between evidence values close to 0. Exemplar-specific attention provided the best overall approximation of the data. Exemplar-specific specificity provided a slightly worse approximation, but it predicted better the rule-like generalization pattern observed.
\end{abstract}

For a long time, perceptual categorization has been the domain of single-system models that assume the existence of a unique representation upon which categorical decisions are made (Ashby \& Gott, 1988; Hintzman, 1986; Nosofsky, 1986; Posner \& Keele, 1968; Reed, 1972). Recently, however, several researchers have identified phenomena that they argue can only result from the interaction between two distinct types of category representations: an abstract "explicit" representation, in which categories are usually represented by simple rules, and an "implicit" representation, formed either by exemplars or by complex rules (Ashby, Alfonso-Reese, Turken, \& Waldron, 1998; Erickson \& Kruschke, 1998; Minda \& Smith, 2001). The debate over single- versus multiple-system models is far from resolved, because many of the empirical findings previously identified as multiple-system phenomena have received single-system explanations as well (Nosofsky \& Johansen, 2000; Nosofsky \& Kruschke, 2002; Nosofsky \& Zaki, 2002).

Exemplar models are a very successful class of singlesystem models (Kruschke, 1992; Nosofsky, 1986, 1987, 1989). According to these models, subjects store individual category exemplars in memory and classify new stimuli in terms of their relative similarities to the stored exemplars. Exemplar models have been able to account for human performance in many distinct conditions, from ill-defined categories (McKinley \& Nosofsky, 1995) to rule-like (Nosofsky, 1987, 1989; Nosofsky, Clark, \& Shin, 1989) and prototype-like (Shin \& Nosofsky, 1992) categories.

Recently, Erickson and Kruschke (2002b) challenged the generality of exemplar models with respect to human category learning. They tested subjects in a rule-plusexception categorization task. Most of the stimuli could be classified correctly by following a simple rule. How- ever, some of the stimuli, the exceptions, violated the rule. Subjects who learned to correctly classify all training stimuli tended to classify new stimuli in a rule-like way, even when these were more similar to the exceptions than to the remaining training stimuli. With the help of model analyses, Erickson and Kruschke (2002b) concluded that exemplar models could not explain adequately the pattern of generalization evident in learners' performance, whereas a multiple-system model could. Here, we readdress the ability of exemplar models to explain human performance in this task.

Previous research has shown that exception stimuli are recognized better than rule-following stimuli after a ruleplus-exception task (Palmeri \& Nosofsky, 1995; Sakamoto \& Love, 2004). This finding has recently received an exemplar explanation (Sakamoto, Matsuka, \& Love, 2004). Commonly, exemplar models assume similarity to be exemplar independent, so that similarity to an exception is judged in the same way as similarity to a rule-following exemplar. Under such an assumption, exemplar models are unable to predict the better recognition for exception stimuli. Sakamoto et al. (2004) extended an exemplar model with exemplar-specific attention and showed that this model could predict better recognition for the exception stimuli. Exemplar-specific attention allows some features of the stimuli to be used when judging similarity to a rule-following exemplar and, simultaneously, other features to be used when judging similarity to an exception. This is precisely what they found when simulating human performance using an extended exemplar model. We expect exemplar-specific attention to succeed in replicating the main findings of Erickson and Kruschke (2002b).

A possible alternative explanation to Erickson and Kruschke's (2002b) findings goes by the name of exemplar- 
specific "specificity." In contrast to exemplar-specific attention, the same stimulus features are considered while judging the similarity to any of the exemplars. Similarity, however, is made exemplar dependent by allowing different exemplars to develop different gradients of similarity. For instance, the parents of identical twins are better able to discriminate between their offspring than between other identical twins. The similarity of each of their own children has a steeper gradient than the similarity of other identical twins. This difference in gradients may be due to increased experience with some stimuli with respect to others, as suggested by Nosofsky $(1987,1991)$. A compelling alternative hypothesis relates exemplar "relevance" to the gradient of similarity: The more relevant an exemplar is, the steeper its gradient of similarity becomes. In a rule-plus-exception task, exceptions are more "relevant" than rule-following stimuli, and therefore might develop steeper gradients of similarity. As a result, stimuli closer to the exceptions do not necessarily need to be classified in the category of the exceptions.

This article is structured as follows. First, we present the exemplar model and its augmented versions. Thereafter, we compare the ability of the augmented versions to explain the data observed in Erickson and Kruschke's (2002b) experiment relative to a traditional exemplar model and a multiple-system model. We conclude with a general discussion.

\section{OVERVIEW OF EXEMPLAR MODELS}

\section{ALCOVE}

In the analyses presented in this article, we make use of the ALCOVE model, introduced by Kruschke (1992). ALCOVE is a feed-forward connectionist network whose basic computations closely mimic those of the generalized context model (GCM; Nosofsky, 1986). The model receives stimuli as input and produces probabilities as output. As in the GCM, the probability of classifying a stimulus into a category depends solely on its similarity to the exemplars stored in the model. In contrast with the GCM, the associations between exemplars and categories are learned, and the same holds for the dimensional attention strengths (see below). Here, we present the version of ALCOVE used within ATRIUM (Erickson \& Kruschke, 1998; Erickson \& Kruschke, 2002a), a multisystem model of category learning. This version differs from the original one (Kruschke, 1992) only in the teacher values used in learning: 0 and 1 , instead of -1 and 1 .

In ALCOVE, stimuli are represented as points in a multidimensional psychological space. Each node in the input layer represents one dimension in the space. Its activity, $d_{i}$, is the coordinate of the stimulus along that dimension. The nodes in the hidden layer represent the exemplars. Let $\left(\mathrm{e}_{j 1}, \ldots, \mathrm{e}_{j n}\right)$ be the positions of the exemplars associated with node $j$. The activity of the node is given by

$$
a_{j}=\exp \left(-.5 c \sum_{i} \alpha_{i}\left|e_{j i}-d_{i}\right|\right),
$$

where $c$ is the specificity of the node, $\alpha_{i}$ the dimensional attention strength of dimension $i$, and $d_{i}$ the coordinate of the stimulus on dimension $i$. In conceptual terms, the activity of an exemplar node corresponds to the similarity between the exemplar it represents and the stimulus. In the output layer, each node represents one category. The activity of an output node is given by a weighted sum of the activities of the exemplar nodes,

$$
a_{K}=\sum_{j} w_{K j} a_{j},
$$

where $w_{K j}$ is the weight of the connection between exemplar node $j$ and category node $K$, and $a_{j}$ is the activity of exemplar node $j$. The activities of the category nodes are converted into probabilities according to the following equation:

$$
p(K)=\frac{\exp \left(\varphi a_{K}\right)}{\sum_{M} \exp \left(\varphi a_{M}\right)},
$$

where $\varphi$ is a response scaling factor that represents the degree of response decisiveness. A high $\varphi$ enhances differences in the activations, resulting in more extreme probabilities, whereas a low $\varphi$ has the opposite effect, blurring those differences.

As stated above, the weights of the connections between exemplar and category nodes, as well as the dimensional attention strengths, are learned. Learning is achieved by gradient descent on the sum-squared error. During each learning episode, the presentation of a stimulus is followed by feedback, indicating the correct category. Feedback is coded in the network by "humble teacher values" (Kruschke, 1992),

$$
t_{K}= \begin{cases}\max \left(1, a_{K}\right) & \text { if the stimulus is in category } K, \\ \min \left(0, a_{K}\right) & \text { otherwise, }\end{cases}
$$

where $a_{K}$ is the activity of category node $K$. Gradient descent on error yields the following learning equations:

$$
\begin{gathered}
\Delta w_{K j}=\lambda_{w}\left(t_{K}-a_{K}\right) a_{j}, \\
\Delta \alpha_{i}=-\lambda_{\alpha} \sum_{j}\left(\sum_{K}\left(t_{K}-a_{K}\right) w_{K j}\right) a_{j} c\left|e_{j i}-d_{i}\right|,
\end{gathered}
$$

where the $\lambda s(\lambda>0)$ are the learning rates. The dimensional attention strengths are constrained to be nonnegative, and so are set to 0 whenever Equation 6 drives them to negative values.

ALCOVE has four free parameters: the specificity of the exemplar nodes $c$, the response scaling constant $\varphi$, the association learning rate $\lambda_{w}$, and the attention learning rate $\lambda_{\alpha}$. In all the simulations reported in this article, the weights between exemplar and category nodes are set initially to 0 , and the dimensional attention strengths $\alpha_{i}$ are set to $1 / n$, where $n$ is the number of dimensions of the psychological space.

\section{ES-ALCOVE}

Sakamoto et al. (2004) have presented a version of ALCOVE with exemplar-specific attention (ES-ALCOVE). This model shares Equations 2-5 with ALCOVE. In ad- 
dition, individual attention weights are incorporated in Equation 1, giving

$$
a_{j}=\exp \left(-.5 c \sum_{i} \alpha_{j i}\left|e_{j i}-d_{i}\right|\right),
$$

where $c$ is the specificity of the node, $\alpha_{j i}$ is the dimensional attention strength for stimulus $j$ along dimension $i$, and $d_{i}$ is the coordinate of the stimulus on dimension $i$. Learning of the dimensional attentional strengths is given by the gradient descent on error:

$$
\Delta \alpha_{j i}=-\lambda_{\alpha}\left(\sum_{K}\left(t_{K}-a_{K}\right) w_{K j}\right) a_{j} c\left|e_{j i}-d_{i}\right|,
$$

where $\lambda_{\alpha}(>0)$ is the attention learning rate. In sum, ESALCOVE is defined by Equations $2-5$ and Equations 7 and 8. Despite the individual dimensional attention strengths, like ALCOVE, it has four free parameters.

\section{ALCOVEc}

In ALCOVE, all exemplars share the same specificity value $c$-that is, the same similarity gradient. To allow each exemplar to develop its own individual gradient, we extend ALCOVE with exemplar-specific specificity values $c_{j}$, which replace the specificity value $c$ in Equation 1. These values are initially equal to some value $c$, but they may become distinct as a result of learning. The learning rule for the $c_{j}$ s as given by gradient descent on the sumsquared error is as follows:

$$
\Delta c_{j}=-\lambda_{c}\left(\sum_{K}\left(t_{K}-a_{K}\right) w_{K j}\right)\left(\sum_{i} \alpha_{i}\left|e_{j i}-d_{i}\right|\right) a_{j},
$$

where $\lambda_{c}$ is the specificity learning rate. The specificity values are constrained to be nonnegative, and so are set to 0 whenever Equation 7 drives them to negative values. Note that the $c_{j}$ values are calculated only on the basis of error values with respect to the correct reproduction of the category measure. They are not parameters that can be freely adjusted in order to increase the fit to the empirical data (i.e., the subjects' performance). The augmented model, ALCOVEc, has five free parameters in total: the four free parameters of ALCOVE and the specificity learning rate $\lambda_{c}$.

\section{A RULE-PLUS-EXCEPTION TASK: ERICKSON AND KRUSCHKE (2002B)}

Erickson and Kruschke (2002b) presented an experiment in which subjects had to classify stimuli into one of six categories. The stimuli were fixed-width rectangles that varied in height and in the horizontal position of an interior vertical line segment. A schematic representation of the stimuli and the respective categories is presented in Figure 1. The great majority of the stimuli can be classified in terms of a simple rule: Stimuli whose inner vertical line is within the left half of the rectangle belong to Category A, whereas the remaining stimuli belong to Category B. However, there are four exceptions to this rule, each one belonging to a distinct category. Along with the training stimuli, there were also transfer stimuli to which no correct category was associated. Erickson and Kruschke (2002b) identified $T_{\mathrm{C}}$ and $T_{\mathrm{F}}$ as transfer stimuli whose pattern of classification would reveal the nature of the representations used in categorization decisions. If subjects were using rules, the critical transfer stimuli $T_{\mathrm{C}}$ and $T_{\mathrm{F}}$ should be classified mainly as members of Categories A and B, respectively. However, if subjects made use of an exemplar representation, $T_{\mathrm{C}}$ and $T_{\mathrm{F}}$ should be classified as members of Categories $\mathrm{C}$ and F, respectively, because of their high similarity to the exception stimuli.

The experiment was carried out in three distinct phases. First, only the exception stimuli were presented; thereafter, all training stimuli; and in the final phase, all training and transfer stimuli. Corrective feedback was given after every training stimulus's presentation. Only the data from the last phase, the transfer phase, were considered in the analyses. The results confirmed the rule representation hypothesis: Subjects who learned to classify all training stimuli above chance (learners) classified stimuli $T_{\mathrm{C}}$ and $T_{\mathrm{F}}$ more often into Categories A and B $(M=.725)$ than into Categories $\mathrm{C}$ and $\mathrm{F}(M=.194)$.

Erickson and Kruschke (2002b) fitted ALCOVE and ATRIUM (Erickson \& Kruschke, 1998, 2002a) to the performance of the learners. ATRIUM, as presented in Erickson and Kruschke (2002a), is a feed-forward connectionist network that learns the correct category for a set of stimuli by error-driven learning. It contains an ex-

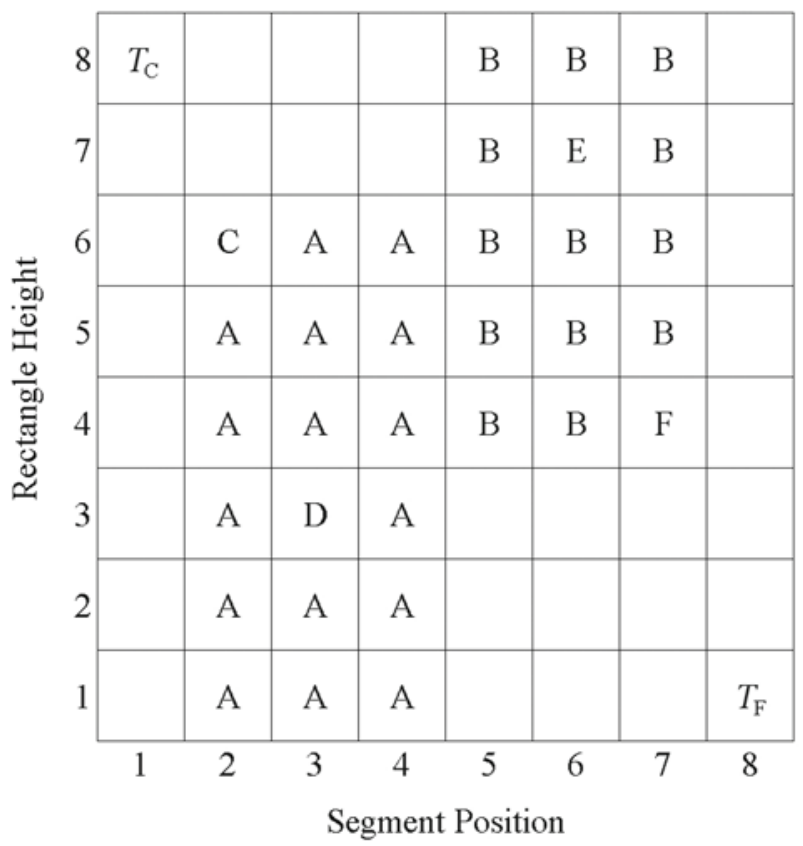

Figure 1. The category structure used by Erickson and Kruschke (2002b). The rows represent the stimulus values for rectangle height, and the columns represent the stimulus values for inner segment position. Cells containing the letters A-F identify training stimuli and their respective categories. Empty cells and the cells containing $T_{\mathrm{C}}$ and $\boldsymbol{T}_{\mathrm{F}}$ identify transfer stimuli. Adapted from "Rule-Based Extrapolation in Perceptual Categorization" by M.A. Erickson and J. K. Kruschke, 2002, Psychonomic Bulletin \& Review, 9, p. 162. Copyright 2002 by the Psychonomic Society, Inc. 
emplar module that works just like ALCOVE. In addition, it has a number of rule modules, one per stimulus dimension. Each rule module contains rules defined on the associated dimension that, through learning, are associated differentially with each category. A gating mechanism learns which module better predicts the category for each exemplar. As a result, some exemplars may be classified more in terms of the exemplar module, whereas others are classified more in terms of the rule modules.

ATRIUM is considerably more complex than ALCOVE, and this complexity is reflected in the number of free parameters, 11 instead of 4 . In addition, ATRIUM contains ALCOVE as a special case, and therefore always performs better. To compare models with different numbers of parameters, we use Akaike's information criterion statistic (AIC; Akaike, 1974). The AIC penalizes models for their numbers of free parameters, allowing those with fewer free parameters to have better (lower) AIC values than those with more free parameters. As auxiliary measures of fit, we also report the root-mean-square deviation (RMSD) and the proportion of variance accounted for $\left(R^{2}\right)$.

Erickson and Kruschke (2002b) found that ALCOVE fitted the data much worse than ATRIUM (see Table 1). Moreover, ALCOVE was not able to correctly predict the pattern of performance observed for the critical stimuli, classifying them mostly in terms of the nearest exception. In contrast, ATRIUM classified these stimuli mostly in terms of a rule. Erickson and Kruschke (2002b) also considered extended versions of ALCOVE, but despite the improvement in fit, none of the fitted models could predict rule-like generalization for the critical transfer stimuli. The researchers concluded that exemplar models

Table 1

Summary Fits and Generalization Patterns for ALCOVE, a Version of ALCOVE With Exemplar-Specific Specificity (ALCOVEc), a Version of ALCOVE With Exemplar-Specific Attention (ESALCOVE), and ATRIUM to the Learners' Performance in Erickson and Kruschke's (2002b) Experiment

Probability of Generalization

\begin{tabular}{lccccc}
\multicolumn{1}{c}{ Model } & AIC & $R^{2}$ & RMSD & Exception & Rule \\
\hline Transfer Stimuli & & & & & \\
ALCOVE & $1,828.28$ & .635 & .166 & .446 & .248 \\
ALCOVEc & $1,822.22$ & .647 & .163 & .427 & .269 \\
ES-ALCOVE & $1,698.05$ & .690 & .153 & .411 & .268 \\
ATRIUM & $1,173.72$ & .850 & .106 & .234 & .419 \\
ALCOVE(EK)* & $2,624.46$ & .465 & .202 & .682 & .127 \\
ATRIUM(EK)* & $1,454.79$ & .840 & .110 & .201 & .550 \\
All Stimuli & & & & & \\
ALCOVE & $2,889.89$ & .715 & .151 & .414 & .240 \\
ALCOVEc & $2,889.89$ & .715 & .151 & .414 & .240 \\
ES-ALCOVE & $2,725.28$ & .754 & .140 & .403 & .245 \\
ATRIUM & $2,017.80$ & .863 & .104 & .320 & .401 \\
Observed Data & & & & .194 & .725 \\
\hline
\end{tabular}

Note-Lower values of AIC reflect a better fit of the model. AIC $=$ Akaike's information criterion statistic; $R^{2}=$ proportion of variance accounted for; RMSD = root-mean-square deviations. *ALCOVE(EK) and ATRIUM(EK) are the ALCOVE and ATRIUM versions used by Erickson and Kruschke (2002b). were not adequately able to predict human performance in this particular experiment.

\section{Model-Based Analyses}

Erickson and Kruschke (2002b) fitted ALCOVE and ATRIUM exclusively to the performance of learners for the 31 transfer stimuli during the transfer phase. The remaining data were left unaddressed because Erickson and Kruschke (1998) had previously shown that ALCOVE had trouble fitting the training and transfer data simultaneously in similar experiments. We fitted ALCOVE, ES-ALCOVE, ALCOVEc, and ATRIUM (Erickson \& Kruschke, 2002a) to the data on the transfer stimuli and found the best fits by minimizing the AIC value using a simulated annealing algorithm (Goffe, Ferrier, \& Rogers, 1994). For all models, we assumed that only the training stimuli were stored as exemplars. In the case of ATRIUM, we further assumed that every possible rule along each of the two stimulus dimensions was present. The best fits reported were obtained by averaging the predicted probabilities over 1,000 simulated subjects. Each simulated subject was presented with a different random sequence of stimulus presentation, generated in accordance with the experimental procedure.

The best fits and the patterns of generalization for the critical transfer stimuli are presented in the upper half of Table 1. ATRIUM yielded the best fit to the data. Furthermore, it predicted more rule-like generalization than exception-like generalization for the critical transfer stimuli, replicating qualitatively the observed performance. All exemplar models yielded considerably worse fits. Neither of the exemplar models was able to replicate the performance observed for the critical transfer stimuli, predicting instead more exception-like generalization than rule-like generalization.

For the sake of completeness, we fitted all models to human performance for both the transfer and training stimuli during the transfer phase. The best fits and patterns of generalization for critical transfer stimuli are presented in the lower half of Table 1. As before, ATRIUM yielded the best fit, followed by ES-ALCOVE, ALCOVEc, and ALCOVE. ATRIUM predicted higher rule-like than exemplar-like generalization for the critical transfer stimuli. None of the exemplar models was able to make this prediction. Despite correctly predicting the pattern of generalization for the critical transfer stimuli, ATRIUM remains far from providing a good quantitative fit. It predicts a difference of .080 between the probability of rule-like generalization and the probability of exemplar-like generalization, whereas the empirical difference is .531 (see Table 1).

\section{Model-Based Analyses Using Power Decisiveness}

ALCOVE and the extended versions we are considering here use Equation 3 to convert the activity of category nodes into probabilities. In this choice rule, response decisiveness is obtained by applying an exponential function to the activities of the category nodes. An alternative formalization of decisiveness has been used in recent for- 
mulations of the GCM model (Ashby \& Maddox, 1993; Nosofsky \& Zaki, 2002):

$$
p(K)=\frac{a_{K}^{\varphi}}{\sum_{M} a_{M}^{\varphi}},
$$

where $\varphi$ is the response scaling parameter. Instead of an exponential function, a power function is used. This change produces, in general, identical results. However, this is not the case when the activity of the category nodes is low. As an example, consider the case of two categories, A and B. Let a stimulus produce category node activities .01 and .001 for Categories A and B, respectively. Assuming $\varphi=1$, exponential decisiveness predicts $p(\mathrm{~A})=.502$ and $p(\mathrm{~B})=.498$, whereas power decisiveness predicts $p(\mathrm{~A})=.909$ and $p(\mathrm{~B})=.091$. It is possible to enlarge the difference between $p(\mathrm{~A})$ and $p(\mathrm{~B})$ under exponential decisiveness by increasing the value of $\varphi$, but at the cost of losing sensitivity for large differences. To our knowledge, both decisiveness functions have been used without any preference. Here, we consider the effect of switching between them for the task and models we have been considering. Power decisiveness only works for nonnegative activation values. However, because of the humble teacher, negative values can occur for any of the models considered. To minimize this problem, we considered a strict teacher instead. In the cases in which category nodes show negative activations, we reset these to 0 .

We incorporated power decisiveness and a strict teacher in all models and fitted them to the data. The best fits and patterns of generalization are presented in Table 2. All exemplar models benefited from the change. In particular, ALCOVEc and ES-ALCOVE benefited the most, now yielding a reasonably good fit to the data $\left(R^{2}>.820\right)$. Furthermore, both models were able to predict qualita-

Table 2

Summary Fits and Generalization Patterns for ALCOVE, a Version of ALCOVE With Exemplar-Specific Specificity (ALCOVEc), a Version of ALCOVE With Exemplar-Specific Attention (ESALCOVE), and ATRIUM to the Learners' Performance in Erickson and Kruschke's (2002b) Experiment, Using Power Decisiveness

\begin{tabular}{lccccc}
\hline & & & & \multicolumn{2}{c}{$\begin{array}{c}\text { Probability of } \\
\text { Generalization }\end{array}$} \\
\cline { 5 - 6 } Model & AIC & $R^{2}$ & RMSD & Exception & Rule \\
\hline Transfer Stimuli & & & & & \\
ALCOVE & $1,609.21$ & .652 & .162 & .643 & .287 \\
ALCOVEc & $1,133.99$ & .848 & .107 & .174 & .677 \\
ES-ALCOVE & $1,049.63$ & .858 & .104 & .333 & .471 \\
ATRIUM & $1,352.11$ & .759 & .135 & .429 & .332 \\
All Stimuli & & & & & \\
ALCOVE & $2,792.75$ & .716 & .150 & .708 & .229 \\
ALCOVEc & $2,066.48$ & .820 & .120 & .281 & .594 \\
ES-ALCOVE & $1,938.97$ & .838 & .113 & .420 & .477 \\
ATRIUM & $2,357.23$ & .808 & .124 & .346 & .412 \\
Observed Data & & & & .194 & .725 \\
\hline
\end{tabular}

Note-Lower values of AIC reflect a better fit of the model. AIC $=$ Akaike's information criterion statistic; $R^{2}=$ proportion of variance accounted for; RMSD = root-mean-square deviations. tively the pattern of generalization observed for the critical transfer stimuli. In this respect, ALCOVEc yielded a better approximation of the data than ES-ALCOVE. It approximated the observed pattern of generalization when fitted to the partial data set, and still did well when fitted to the complete data set. In both cases, the differences between rule-like and exception-like probabilities of generalization was greater than 310 . ES-ALCOVE predicted a smaller difference, particularly with the full data set (.057). ALCOVE, on the other hand, despite showing a slight improvement in the fits, was still unable to predict the correct pattern of generalization. We conclude that the simple substitution of the decisiveness function is not sufficient to allow exemplar models to predict rule-like generalization for the critical transfer stimuli. Instead, the addition of exemplar-specific specificity or the addition of exemplar-specific attention is necessary.

In contrast with the exemplar models, ATRIUM showed worse fits. Furthermore, it did not predict the pattern of generalization observed when fitted to the transfer stimuli. A comparison between the best version of ATRIUM (i.e., the one using exponential decisiveness) and the best versions of ALCOVEc and ES-ALCOVE (i.e., the ones using power decisiveness) shows that all models yielded almost identical fits, with ES-ALCOVE yielding slightly better ones. All models were able to replicate qualitatively the pattern of generalization for the critical transfer stimuli. ALCOVEc, however, yielded the best approximation. A closer look at ALCOVEc shows that all rule-consistent exemplars developed a lower specificity value than the exception exemplars, and as a result, whenever stimulus $T_{\mathrm{C}}$ or $T_{\mathrm{F}}$ was presented, the closest exemplar node representing the rule-consistent training stimuli had the highest activation. Because these exemplars were associated mostly with Category A or B, the corresponding category node showed the highest activation. These activation values were small, though, so it was essential to use power decisiveness to transform the small differences in activation into larger differences in probabilities.

\section{DISCUSSION}

In a previous test, Erickson and Kruschke (2002b) argued that exemplar models could not properly fit human performance in a rule-plus-exception categorization task. In contrast, ATRIUM (Erickson \& Kruschke, 1998, 2002a), a multiple-system model, could. In particular, it could predict rule-like generalization for a set of critical stimuli. Here, we amend this conclusion by showing that certain exemplar models can fit the data as well as ATRIUM and, simultaneously, reproduce rule-like generalization. We considered two distinct extensions of exemplar models: exemplar-specific attention and exemplarspecific specificity. The former has recently been used to explain better recognition of exception stimuli in ruleplus-exception tasks (Sakamoto et al., 2004). The latter has been suggested in previous research but not yet used in the rule-plus-exception context. None of these models provided improved fits with respect to ATRIUM or replicated rule-like generalization. This was only possible 
after substituting a power function for the exponential decisiveness function in the choice rule. To our knowledge, previous research has not fully explored the consequences of such a change. Although the extension using exemplarspecific attention provided the best explanation of the data, exemplar-specific specificity provided the most accurate replication of rule-like generalization, and its fit still approximated that of ATRIUM.

The extended exemplar models were able to produce rule-like generalization, but perhaps at the cost of highly specific parameter values. We explored this possibility by performing a grid search on the parameter values. We found that the specificity learning rate $\left(\lambda_{c}\right)$ and the initial specificity $(c)$ parameters were the most critical ones in ALCOVEc. In general, rule-like generalization varies proportional to $\lambda_{c}$ and inversely proportional to $c$. The ALCOVEc model predicts rule-like generalization if $\lambda_{c}$ is high enough and $c$ is not too high. In ES-ALCOVE, the initial specificity $(c)$ and the attention learning rate $\left(\lambda_{\alpha}\right)$ are the critical parameters, and rule-like generalization is obtained when $\lambda_{\alpha}$ is large enough and $c$ is not too low or too high. Outside the ranges specified, both models mostly predict exception-like generalization. In sum, both are able to predict robustly rule-like generalization.

Why was ES-ALCOVE unable to replicate the pattern of generalization as well as ALCOVEc? Both models classify the critical transfer stimuli in terms of the closest rulelike exemplars. However, in ES-ALCOVE, some of these exemplars develop a stronger association with the exception category than with the correct category, but are nonetheless categorized correctly because of the contribution of the surrounding exemplars, which share their category and one value along one of the dimensions. Stimulusspecific attention brings the neighboring exemplars together, allowing correct categorization of those exemplars with exception-like associations. The end result, however, is a smaller difference between rule-like and exceptionlike generalization for the critical transfer stimuli.

Erickson and Kruschke (1998) reported an experiment similar to the one analyzed here. Their main result was identical: Transfer stimuli closer to an exception than to the rule-following exemplars were classified mostly according to the rule. Erickson and Kruschke (1998) fitted ES-ALCOVE to the data, but it was unable to predict this generalization pattern very well. Our results show that their result may have been due to the use of exponential decisiveness in the choice rule, and that power decisiveness may provide a better approximation.

We have focused our analysis on exemplar models as a single-system explanation for human performance in a rule-plus-exception task. However, we do not rule out that other single-system models, such as SUSTAIN (Sakamoto $\&$ Love, 2004), may perform as well or even better. Further research is needed to test this possibility. Our results, along with previous ones (Sakamoto et al., 2004), indicate that exemplar models provide a good approximation of human performance in the ecologically interesting class of rule-plus-exception tasks.

\section{AUTHOR NOTE}

This research was supported by Grant SFRH/BD/8614/2002 to P.M.R. from the Portuguese Foundation for Science and Technology (FCT). We thank Michael Erickson for providing us with the original data and best-fit predictions obtained for the experiment reported in Erickson and Kruschke (2002b). Correspondence concerning this article should be addressed to either P. M. Rodrigues or J. M. J. Murre, Department of Psychology, University of Amsterdam, Roeterstraat 15, 1018 WB Amsterdam, The Netherlands (e-mail: p.m.deandraderodrigues@uva.nl or jaap@murre.com).

\section{REFERENCES}

AKaIKE, H. (1974). A new look at the statistical model identification. IEEE Transactions on Automatic Control, 19, 716-723.

Ashby, F. G., Alfonso-Reese, L. A., Turken, A. U., \& Waldron, E. M. (1998). A neuropsychological theory of multiple systems in category learning. Psychological Review, 105, 442-481.

Ashby, F. G., \& GotT, R. E. (1988). Decision rules in the perception and categorization of multidimensional stimuli. Journal of Experimental Psychology: Learning, Memory, \& Cognition, 14, 33-53.

AshBY, F. G., \& MADDOX, W. T. (1993). Relations between prototype, exemplar, and decision bound models of categorization. Journal of Mathematical Psychology, 37, 372-400.

Erickson, M. A., \& KruschKe, J. K. (1998). Rules and exemplars in category learning. Journal of Experimental Psychology: General, 127, 107-140.

ERICKSON, M. A., \& KruschKe, J. K. (2002a). Multiple representations in inductive category learning: Evidence of stimulus- and taskdependent representation. Manuscript submitted for publication.

ERICKSON, M. A., \& KruschKe, J. K. (2002b). Rule-based extrapolation in perceptual categorization. Psychonomic Bulletin \& Review, 9, 160-168.

Goffe, W. L., Ferrier, G. D., \& Rogers, J. (1994). Global optimization $\mathrm{C}$ of statistical functions with simulated annealing. Journal of Econometrics, 60, 65-99.

HiNTZMAN, D. L. (1986). "Schema abstraction" in a multiple-trace memory model. Psychological Review, 93, 411-428.

KruschKe, J. K. (1992). ALCOVE: An exemplar-based connectionist model of category learning. Psychological Review, 99, 22-44.

McKinley, S. C., \& Nosofsky, R. M. (1995). Investigations of exemplar and decision bound models in large, ill-defined category structures. Journal of Experimental Psychology: Human Perception \& Performance, 21, 128-148.

MiNDA, J. P., \& SMITH, J. D. (2001). Prototypes in category learning: The effects of category size, category structure, and stimulus complexity. Journal of Experimental Psychology: Learning, Memory, \& Cognition, 27, 775-799.

NoSOFSKY, R. M. (1986). Attention, similarity, and the identificationcategorization relationship. Journal of Experimental Psychology: General, 115, 39-57.

NoSOFSKY, R. M. (1987). Attention and learning processes in the identification and categorization of integral stimuli. Journal of Experimental Psychology: Learning, Memory, \& Cognition, 13, 87-108.

NosOFSKY, R. M. (1989). Further tests of an exemplar-similarity approach to relating identification and categorization. Perception \& Psychophysics, 45, 279-290.

NOSOFSKY, R. M. (1991). Tests of an exemplar model for relating perceptual classification and recognition memory. Journal of Experimental Psychology: Human Perception \& Performance, 17, 3-27.

Nosofsky, R. M., Clark, S. E., \& Shin, H. J. (1989). Rules and exemplars in categorization, identification, and recognition. Journal of Experimental Psychology: Learning, Memory, \& Cognition, 15, 282-304.

NoSOFSKY, R. M., \& JOHANSEn, M. K. (2000). Exemplar-based accounts of "multiple-system" phenomena in perceptual categorization. Psychonomic Bulletin \& Review, 7, 375-402.

NosOFSKY, R. M., \& KRUSCHKE, J. K. (2002). Single-system models and interference in category learning: Commentary on Waldron and Ashby (2001). Psychonomic Bulletin \& Review, 9, 169-174.

NosofsKy, R. M., \& ZAKI, S. R. (2002). Exemplar and prototype models revisited: Response strategies, selective attention, and stimulus gen- 
eralization. Journal of Experimental Psychology: Learning, Memory, \& Cognition, 28, 924-940.

PALMERI, T. J., \& NOSOFSKY, R. M. (1995). Recognition memory for exceptions to the category rule. Journal of Experimental Psychology: Learning, Memory, \& Cognition, 21, 548-568.

PoSNER, M. I., \& KeELE, S. W. (1968). On the genesis of abstract ideas. Journal of Experimental Psychology, 77, 353-363.

REED, S. K. (1972). Pattern recognition and categorization. Cognitive Psychology, 3, 382-407.

SAKAMOTO, Y., \& LOVE, B. C. (2004). Schematic influences on category learning and recognition memory. Journal of Experimental Psychology: General, 133, 534-553.
Sakamoto, Y., Matsuka, T., \& Love, B. C. (2004). Dimension-wide vs. exemplar-specific attention in category learning and recognition. In M. Lovett, C. Schunn, C. Lebiere, \& P. Munro (Eds.), Proceedings of the 6th International Conference on Cognitive Modeling (pp. 261266). Mahwah, NJ: Erlbaum.

ShIN, H. J., \& Nosofsky, R. M. (1992). Similarity-scaling studies of dot-pattern classification and recognition. Journal of Experimental Psychology: General, 121, 278-304.

(Manuscript received March 23, 2006; revision accepted for publication August 18, 2006.) 